\title{
Effect of corrosion on time-dependent reliability of steel sheet-pile seawalls in marine environment conditions
}

\author{
H. Yáñez-Godoy, J. Boéro \& G. Thillard \\ Oxand S. A., Avon-Fontainebleau, France
}

F. Schoefs

GeM, University of Nantes, France

\begin{abstract}
Modeling the long term corrosion of steel structures in sea or estuary areas is still a great challenge when analyzing their safety and optimizing their maintenance. The phenomenon of corrosion is very complex due to the nature of the environment and the materials, in addition to the type of the structure and its uses, which all have to be characterized and modeled for performing structural analysis which can integrate the loss of steel thickness. In this paper we present a reliability analysis of one steel sheet-pile seawall considering the effect of corrosion along the depth of the structure. Experimental data to model the corrosion process comes from a great number of ultrasonic residual measurements in some French harbors. A probabilistic model of corrosion is then suggested and is coupled to a 2-D finite element model of the structure to assess the probability of failure at several depths along the steel sheet-pile seawall and for several time periods. Non-intrusive stochastic finite element methods are used for calculations.
\end{abstract}

\section{INTRODUCTION}

One of the main degradation mechanisms of steel marine structures is corrosion. This process is very complex due to the nature of the environment, the material, but also the type of the structure being studied. This physicochemical and biological phenomenon develops randomly in space and time. The loss of thickness which results from corrosion needs to be characterized and modeled for structural analysis. Moreover, due to the randomness of the corrosion process, probabilistic models are needed for structural reliability (Melchers 2009). Ultrasonic residual measurements allow for determining vertical profiles of loss of thickness, identifying the areas which are the most affected by corrosion and stating the assumptions for modeling the random corrosion process. Several inspection campaigns have been performed during the three last decades in some French harbors and thus a great number of ultrasonic residual measurements are now available for structures in marine environment (Boéro et al., 2009a,b).

First, let us focus this paper on the description of the corrosion data base and its use to analyze and model the stochastic field of corrosion. Then, the spatio-temporal corrosion model of steel in marine environments can be developed on the basis of this statistical analysis of corrosion measurements. Finally, the predictions of the model are represented with the polynomial chaos decomposition, which is to be introduced into a reliability analysis that is performed by a means of structural stochastic computations. These computations use spectral stochastic finite element methods and are devoted to study and quantify the change of mechanical behavior influenced by corrosion in marine structures. 


\section{CORROSION DATA BASE}

\subsection{Available data}

During the French project GEROM (Risk Management of maritime and river structures in harbors, 2006-2009), an inquiry was realized with the goal of analyzing the maintenance practices of the owners and to collect ageing data from the most important French harbors (Boéro et al., 2009a,b). This paper focuses on data concerning the corrosion of steel structures like sheet-pile wharves that were built between 1955 and 1985. The characterization of the corrosion rests mainly on the thickness steel measurements done by Non Destructive Techniques (ultrasonic measurements meanly) and began about in 1990 in France as a decision-aid-tool. Thus most of the structures are between 20 and 30 years old at the time of inspection. So in this paper, due to the lack of data during the first years of exposure and in view to compare maintenance strategies in future works, only the long-term of corrosion process is analyzed. Furthermore, we focus on structures located in an environment where tides are present. The variation in water levels for the studied wharves in several harbors extends between 0.5 and 9.1 meters. Environment parameters around these structures are very similar from a corrosion point of view (temperature, nutrients, salinity, ...), we consider that structures are located in the same environment and subjected to the same corrosion mechanisms in each exposure area (tidal zone, immersion zone, mud zone, etc.) (Boéro et al., 2009c, Melchers 2009).

\subsection{Inspection protocol and data pre-processing}

Corrosion of steel structures is classically assessed by ultrasonic non destructive testing. In France, a protocol is given by the CETMEF and classically used by several companies to perform inspection planning of coastal marine steel structures. The CETMEF is a French governmental office devoted to the diffusion of knowledge, to provide technical and research studies as well as engineering and expert's reports. This protocol consists in performing residual thickness measurements for several depths of the structure using ultrasonic testing after removing corrosion products by grinding. By using this technique and considering the harsh conditions for marine inspections, the error on the measurements at a given point on the structure cannot be neglected. Error comes both from the physical measurement (around $0.1 \mathrm{~mm}$ ) and from the protocol (grinding, link diver-operator, etc.). Thus three measurements are performed on a given location: the three measurements are distributed in an about

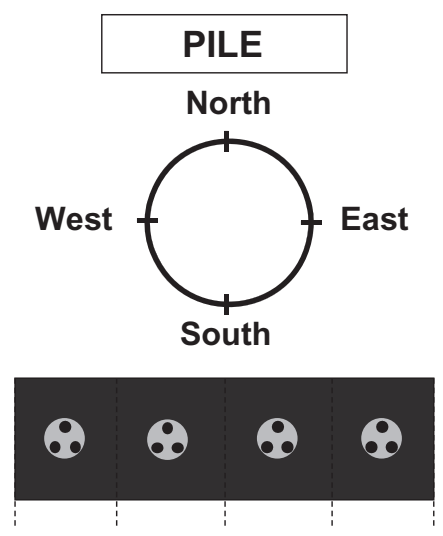

North East South West

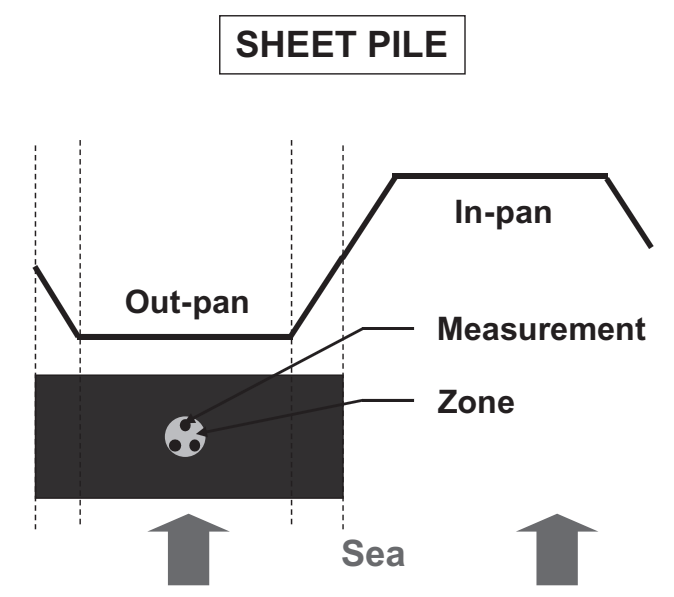

Figure 1. CETMEF protocol: location of inspections for pile (left) \& sheet-pile (right). 
$5 \mathrm{~cm}$ diameter disk, every $120^{\circ}$ as shown in Figure 1. The average of these three readings is considered as the "true" loss of thickness. In fact, it has been shown that this protocol allows filtering the measurement noise (material and human factors) (Schoefs et al., 2009). For on-pile wharves, in view to analyze the corrosion around the pile, measurements are performed at several cardinal positions around the pile section. For on-sheet-pile-wall wharves, they are carried out along the length of the wharf approximately every 10 meters, which is classically the mean distance between two piles for on-pile wharves. Starting from these residual thickness values, and knowing the initial thickness of piles or sheet-piles, we deduce the thickness loss mainly due to corrosion.

\section{ANALYSIS AND MODELING OF THE STOCHASTIC FIELD OF CORROSION}

The loss of thickness due to corrosion is considered here as a stochastic field indexed by the spatial coordinates and the time $t$. Due to the corrosion process, we consider that the principal directions are known: $x$, along the wharf length and $z$, along the wharf depth.

Starting from the analysis of the second-order stationary corrosion process in $\mathbf{R}^{2}$, i.e. according to the two coordinates $x$ and $z$ presented in (Boéro et al., 2009c), a stochastic modeling of the field of corrosion is based on a statistical analysis of more than 35000 data collected in French harbors. True values have been deduced after removing errors of measurement.

The aim of modeling the corrosion in harbors is to provide predictive models that account for the spatial dependence both in depth and in length and the time variability.

The model relies on the general hypothesis that corrosion is a decoupled phenomena with respect to $x$ and $z$. The corrosion can be considered as uniform in a given zone of exposure (tidal zone, immersion zone, etc.). Thus, the distribution of the corrosion $c(0, z, t, \theta)$ is assumed to be independent of $x$; it evolves with the time $t$ and is discretized with $z$ : it depends on the zone of exposure $z_{j}$. The spatial characteristics of the corrosion in the $x$ direction are represented by a deterministic tendency $T\left(x, z_{\vec{j}}, t\right)$. Since no spatial correlations have been made (less than $20 \mathrm{~cm}$ ), the random variables are considered to be independent between two points in the $x$ direction (Boéro et al., 2009c). Finally, the corrosion model can be written in the following form:

$$
c\left(x, z_{\vec{j}}, t, \theta\right)=T\left(x, z_{\vec{j}} t\right)+c\left(0, z_{\vec{j}}, t, \theta\right)
$$

where: $c\left(x, z_{j}, t, \theta\right)$ is loss of thickness in the $\mathrm{x}$-direction, over the time, $t$, for the zone of exposure $z_{j}(\mathrm{~mm})$;

$T\left(x, z_{i}, t\right)$ is the central deterministic tendency of the corrosion field in the $x$ direction;

$c\left(0, z_{i}, t, \theta\right)$ is a random variable which represents the loss of thickness with respect to time $t$ for the zone of exposure $z_{j}(\mathrm{~mm})$.

The Gamma probability distribution is used for modeling the variability of the corrosion. This corrosion probabilistic modeling provides the best estimation to real life situations. Its parameters, $\alpha$ and $\beta$, respectively represent the shape and the scale of the distribution and depend directly on the average and standard deviation of the loss of thickness. These parameters are spatially and time dependent. A statistical analysis of the empirical data for each zone of exposure allowed for the adjustment, by the method of least squares, of the evolution of the mean and the standard deviation of the loss of thickness (Figures 2-3).

In coherence with literature, the average loss of thickness is maximum in zone of low seawater level due to the differential aeration which is established between the tidal zone, very oxygenated, and the immersion zone (Memet 2000).

The distributions of the loss of thickness obtained from the corrosion model after an exposure time of 25 years are illustrated in Figure 4. The predictions of corrosion can thereby be introduced in a reliability analysis, but also in traditional engineering for the revaluation of corroded structures through quantiles for example. 


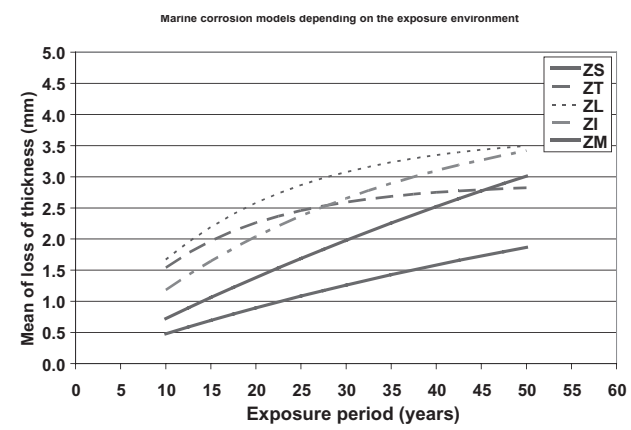

Figure 2. Temporal laws of evolution of mean of loss of thickness.

Legend: $\mathrm{ZS}=$ Splash zone; $\mathrm{ZT}=$ Tidal zone; $\mathrm{ZL}=$ Low seawater level zone; $\mathrm{ZI}=$ Immersion zone; $\mathrm{ZM}=$ Mud zone.

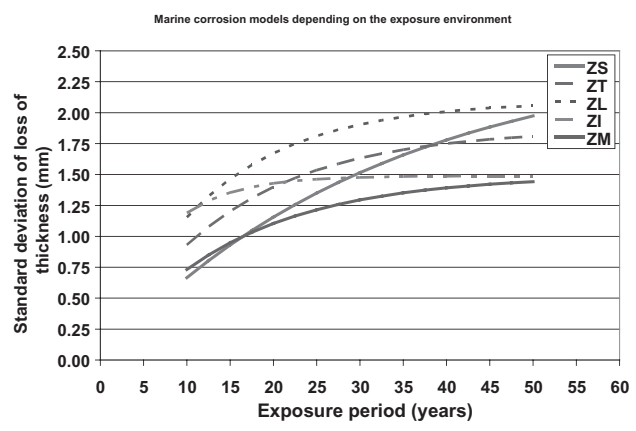

Figure 3. Temporal laws of evolutions of standard deviation of loss of thickness.

Legend: $\mathrm{ZS}=$ Splash zone; $\mathrm{ZT}=$ Tidal zone; $\mathrm{ZL}=$ Low seawater level zone; $\mathrm{ZI}=$ Immersion zone; $\mathrm{ZM}=$ Mud zone.

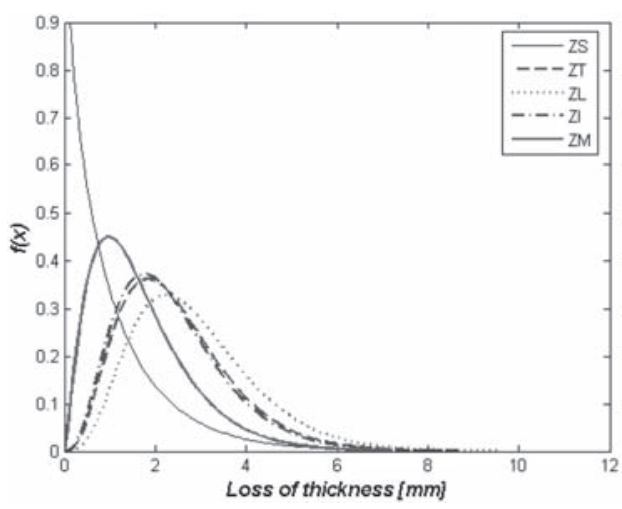

Figure 4. Gamma distributions of loss of thickness for several exposure zones for $t=25$ years. Legend: $\mathrm{ZS}=$ Splash zone; $\mathrm{ZT}=$ Tidal zone; $\mathrm{ZL}$ = Low seawater level zone; $\mathrm{ZI}=$ Immersion zone; $\mathrm{ZM}=$ Mud zone.

\section{MECHANICAL BEHAVIOUR INFLUENCED BY CORROSION IN MARINE STRUCTURES}

\subsection{Example of application: Steel sheet-pile seawall}

This study aims to integrate the spatio-temporal corrosion process that was previously defined in the analysis of the mechanical behavior of a quay using a finite element method. The selected example is representative of current situations and considers a wharf with $U$ shaped piles, anchored through one level of passive tie-rods. The main geometrical and mechanical features of the studied quay are shown in Figure 5.

The quay is long enough for the calculation to be carried out in cross sections (assumption of in-plane deformation). With regards to the interactions between the soil and the water, only the buoyancy forces being exerted on the soil are taken into account. The force of flow that is generated by the viscous friction of the fluid on the grains of the soil is negligible due to the value of $f(9 \mathrm{~m})$. The construction phase of the quay is also not taken into account.

The soil is represented by a homogeneous powder material, with an elastic perfectlyplastic mechanical behavior (criterion of Mohr-Coulomb). The behavior of the steel (main wall, sheet- pile anchorage and tie-rods) is supposed to be purely elastic and isotropic. The elements of interface, laid out around the main wall, are described by Coulomb's friction criterion. The angle of the interface is equal to $2 / 3$ рэ (approximately 20 degrees), and the contact cohesion is negligible. 


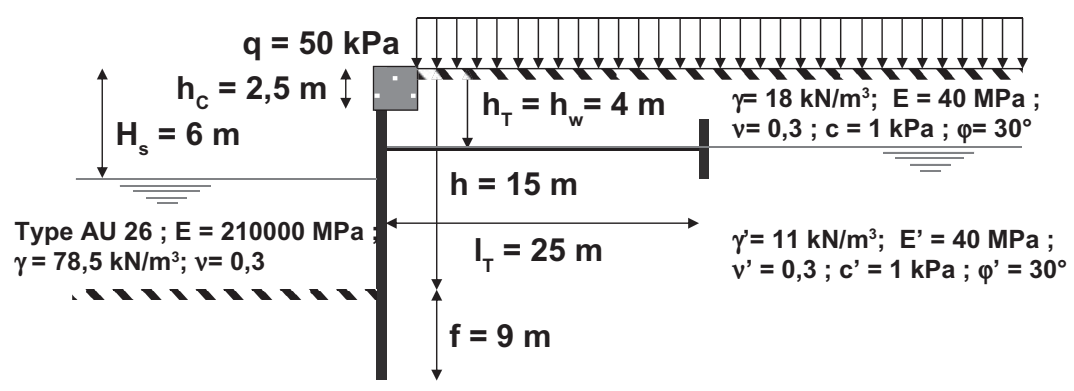

Figure 5. Geometrical and mechanical characteristics of studied structure.

For the studied case, the seawall is topped by a reinforced concrete beam. Therefore, the steel sheet-pile seawall is only in contact with the tidal zone, the zone of low seawater level, the immersion zone and the mud zone. The study is undertaken for 4 different exposure times $(\mathrm{t}=0,10,25$ and 50 years). In a first time, a sensibility analysis of the bending moment to the corrosion is led by means of the spatial evolution of the $95 \%$ fractile of the loss of thickness along the seawall defined by the predictive model (Boéro 2009) (Figure 6). Simulations are carried out using the CAST3M finite elements computer code (http://www.cast3m.cea.fr).

We note the corrosion generates a decrease of the geometrical characteristics of sheetpiles with time and consequently the seawall becomes more flexible. A decrease of bending moment is then observed on the wall towards the seaside excavation (about $15 \%$ after 50 years of exposure in a marine environment). In exchange, the normal strength in the tie-rods increases slightly, $5 \%$ in this case. The bending moments in the zone of embedment and at the level of the passive tie-rods are little affected by corrosion effect. The maximum bending moment is situated in the immersion zone. As a result, the zone where the corrosion is the most severe does not necessarily correspond to the zone which is the most mechanically demanding. These aspects are more developed in the next section where a reliability analysis allows for taking into account the variability of the corrosion.

\section{RELIABILITY ANALYSIS}

\subsection{Polynomial chaos decomposition of the loss of thickness predictions}

The loss of thickness forecasted by the corrosion model at several times and for various zones of exposure is represented with a polynomial chaos (PC) decomposition. This method is chosen to perform structural stochastic computations using spectral stochastic finite element methods (Ghanem \& Spanos 2003).

Thus, each gamma random variable $X$ for each zone of exposure $z_{j}$ can be represented in a Laguerre polynomial basis as follows:

$$
X=\sum_{i=0}^{\infty} a_{i} l_{i}(\xi)
$$

where $\xi$ is a standardized exponential variable defined by $\xi=\phi^{-1} f_{\left.c_{(0, z j, t \theta}\right)}(c \mid \alpha, \beta) ; f c$ is the Gamma probability density function (pdf) of loss of thickness with parameters $\alpha$ and $\beta ; \phi$ is the standardized exponential pdf; $l$ are the Laguerre polynomials which are orthogonal with respect to the joint probability distribution of $\xi$; and $a_{\text {i }}$ are the coefficients to be determined and they are obtained by the projection method (Berveiller 2005). Figure 7 illustrates the distribution of the loss of thickness obtained from the corrosion model after an exposure time of 25 years for each zone $z_{j}$ using PC decomposition. 


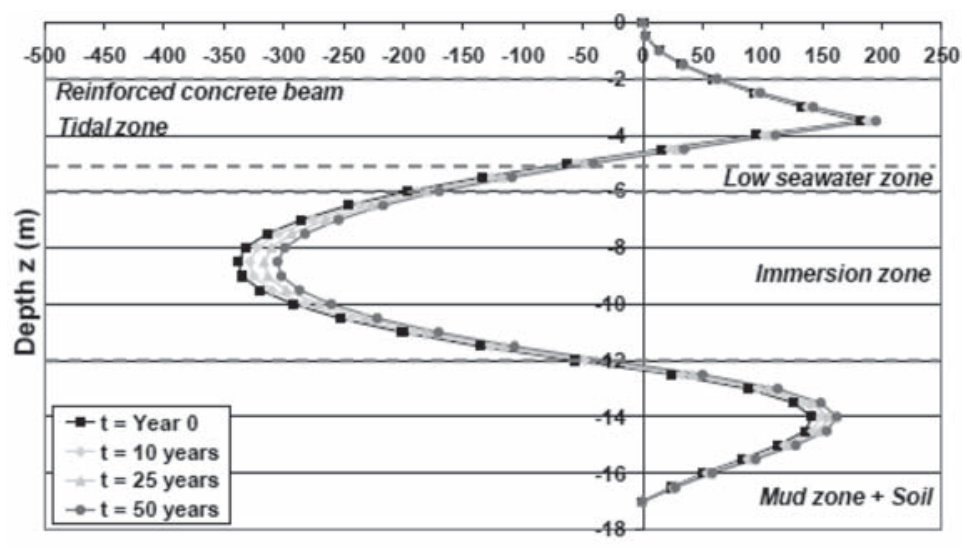

Bending moment (kNm/ml)

Figure 6. Bending moment in the seawall.

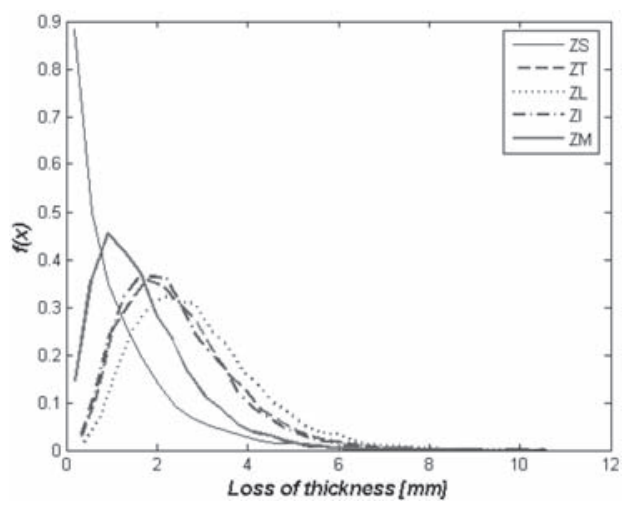

Figure 7. Polynomial chaos decomposition of loss of thickness for several exposure zones for $\mathrm{t}=25$ years. Legend: $\mathrm{ZS}=$ Splash zone; ZT = Tidal zone; ZL = Low seawater level zone; ZI = Immersion zone; $\mathrm{ZM}=$ Mud zone.

\subsection{Assessment of the probability of failure due to corrosion of seawall} for several exposure times

The objective of this section is to compare the evolution of the probability of failure along the seawall considering the following limit state criterion:

$$
G=\sigma_{R}-\sigma_{S_{c(i, z, j, t, \theta)}}(X)
$$

where $\sigma_{R}$ is the maximum initial strength of the steel sheet-pile wall $(t=0)$ and $\sigma_{S c(0, z, j, t, \theta)}(X)$ is the evolution of the stress in the steel sheet-pile wall at time $t$ for each exposure zone $z_{j}$, considering the vector of basic Gamma random variables characterizing the variability of corrosion $X=\left\{X_{1}, X_{2}, \ldots, X_{M}\right\}$; where $M=4$. Note that in some cases, the displacement of the head of the wall can be considered as a limit state too.

Stresses along the depth $z$ are obtained by the determinist 2-D mechanical model, previously presented (same calculation hypothesis as showed in Figure 5 but with an important soil cohesion value are considered). The probability of failure is calculated then as:

$$
P_{f}=P(G<0)=P\left(\sigma_{\left.S c_{[(o, z]}, t, \theta\right]}(X)>\sigma_{R}\right)
$$


Stress $\sigma_{S_{\tau(0, z] j, t, \theta)}}$ can be calculated by an approximation of $\tilde{g}_{S}(X)$ which is defined by:

$$
\tilde{g}_{S}\left(X_{1}\left(\xi_{1}\right), \ldots, X_{M}\left(\xi_{M}\right)\right)=\sum_{j=0}^{P-1} g_{j} H_{j}\left(\left\{\xi_{k}\right\}_{k=1}^{M}\right)
$$

where $\left\{\xi_{I}, \ldots, \xi_{M}\right\}$ are the standardized Gamma variables used to develop every input random variable $X_{l}$, i.e. each gamma random variable, $l \in\{1, \ldots, \mathrm{M}\}$ and $\left\{H_{0}, \ldots, H_{p-l}\right\}$ is the polynomial chaos (PC) basis. Here, the basis functions are chosen to be orthonormal, i.e. $E\left(H_{i} H_{j}\right)=$ $\delta_{i j}$. For a polynomial chaos of degree $p$ in dimension $M$, the number of basis functions is $P=$ $(M+p) !(M ! p !)^{-1}$. The coefficients of the $\mathrm{PC}$ decomposition are defined by the projection method:

$$
g_{j}=E\left(\tilde{g} H_{j}\right)=\int_{R} M \tilde{g}(X(y)) H_{j}(y) \phi_{M}(y) d y
$$

where $\varphi M(y)$ is the multi-gamma probability density function. Integration of the function in Equation (6) can be obtained by using Gaussian quadrature. The Gaussian quadrature formula is written as:

$$
\int_{R} M \tilde{g}(X(y)) H_{j}(y) \phi_{M}(y) d y \approx \sum_{t_{1}=1}^{N} \cdots \sum_{t_{M}=1}^{N} w_{i_{1}} \cdots w_{t_{M}} \tilde{g}\left(X_{1}\left(y_{i_{1}}\right), \cdots, X_{M}\left(y_{i_{M}}\right)\right) H_{j}\left(y_{i_{1}}, \cdots, y_{i_{M}}\right)
$$

where the $\left(y_{1}, \ldots, y_{N}\right)$ and $\left(w_{1}, \ldots, w_{N}\right)$ are the one-dimensional Gauss-Lagrange integration points and weights. These integration points are the $N$ roots of one-dimensional Lagrange polynomial of degree $N$, denoted $h_{N}$. The integration weight $W_{i}$ can be calculated as follows:

$$
W_{t}=\frac{1}{N h_{N-1}\left(y_{t}\right)^{2}} \forall i \in\{1, \ldots, N\}
$$

For each integration point we compute the values of $\left\{X_{1}\left(y i_{1}\right), \ldots, X_{M}\left(y i_{M}\right)\right\}$ assessing their expansion on PC. The number of computations of the deterministic 2-D model is $N^{M}$.

Once coefficients $g_{j}$ in Equation (5) are calculated, evaluation of $P_{f}$ can be performed for the three different exposure times $\left(t=10,25\right.$ and 50 years); the evolution of $P_{f}$ is shown in Figure 8.

We note that the probability of failure is approximately 0.4 at $t=10$ years in the part of the main wall towards the seaside excavation, 0.1 at the level of the passive tie-rods and 0.01 in the zone of embedment. The most critical zone is situated in the immersion zone (ZI).

At $t=50$ years in relation to $t=10$ years, we highlight the progress of the probability of failure due to an increase of loss of thickness in three studied zones: 0.1 to 0.6 at the level of the passive tie-rods; 0.4 to 0.95 in the part of the main wall towards the seaside excavation and 0.01 to 0.25 in the zone of embedment.

\section{CONCLUSIONS}

The spatial variability of steel corrosion in marine environments cannot be objectively described in a quantitative way by deterministic models. Thus, a probabilistic model is suggested to predict stochastic fields of corrosion. This model is based on the experience feedback of the French owners and data available, i.e. measurements of the residual thicknesses of steels by ultrasonic non destructive techniques at several depths and several locations along the French harbor structures during the three last decades.

The predictions which result from these measurements at several times and for various zones of exposure can be represented with a polynomial chaos decomposition with the aim to perform structural stochastic computations using a spectral stochastic finite element method. 


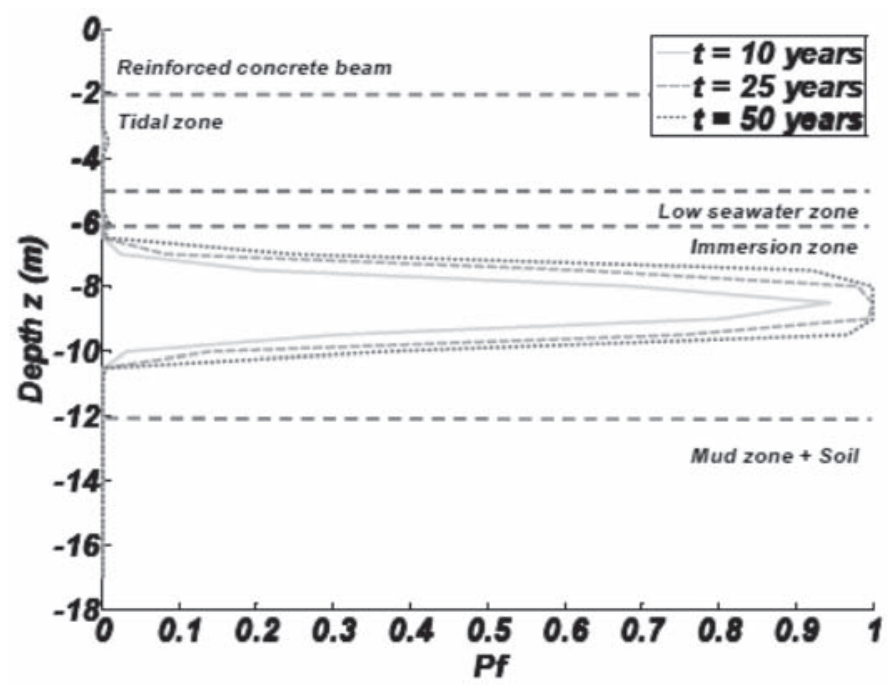

Figure 8. Evolution of the probability of failure along the steel sheet-pile seawall for several exposure times.

This process is used in this paper to lead a time-dependent reliability analysis with the objective of assessing the impact of steel corrosion on the mechanical behavior of a steel sheet-pile seawall.

The complexity of the soil-structure interaction and the influence of corrosion on the mechanical behavior of the steel sheet-pile seawalls are highlighted. The probability of failure, based on a mechanical criterion and deduced using non-intrusive methods, shows two particular zones on the seawall: the part of the main wall towards the seaside excavation and at the level of the passive tie-rods where risks due to corrosion are important. This conclusion brings answers to the owners to help them define optimization inspection plans. This analysis appears to be useful in the re-evaluation of older structures.

\section{REFERENCES}

Berveiller, M. 2005. Éléments finis stochastiques: approches intrusive et non intrusive pour des analyses de fiabilité. Thèse de doctorat. Université Blaise Pascal-Clermont II, Clermont-Ferrand, France. $174 \mathrm{pp}$.

Boéro, J. 2009. Projet GEROM: Gestion par les risques et optimisation de la maintenance des ouvrages portuaires. XXVIIèmes rencontres de l'AUGC, Saint-Malo, 2009.

Boéro, J., Schoefs, F., Capra, B. \& Rouxel, N. 2009a. Risk management of French harbor structures. Part 1: Description of built assets. PARALIA, 12 pp., in press.

Boéro, J., Schoefs, F., Capra, B. \& Rouxel, N. 2009b. Risk management of French harbor structures. Part 2: Current practices, needs-Experience feedback of owners. PARALIA, 12 pp., in press.

Boéro, J., Schoefs, F., Melchers, R. \& Capra, B. 2009c. Statistical Analysis of Corrosion Process along French Coast. 10th Int. Conf. on Structural Safety and Reliability ICOSSAR 2009.

Ghanem, R. \& Spanos, P. 2003. Stochastic finite elements: a spectral approach. Revised edition, New York: Dover.

Melchers, R.E. 2009. Experiments, science and probability theory in building new models for long-term pitting corrosion in marine environments. 10th Int. Conf. on Structural Safety and Reliability ICOSSAR 2009.

Memet, J.B. 2000. La corrosion marine des structures métalliques portuaires: étude des mécanismes d'amorçage et de croissance des produits de corrosion. Thèse de doctorat. Université de La Rochelle, France. 164 pp.

Schoefs, F., Clément, A. \& Nouy, A. 2009. Assessment of spatially dependent ROC curves for inspection of random fields of defects. Structural Safety, in press. 